BULL. AUSTRAL. MATH. SOC.

$46 \mathrm{H} 20,46 \mathrm{~J} 35$

VOL. $23(1981), 479-480$.

\title{
ON SOME GELFAND-MAZUR LIKE THEOREMS \\ IN BANACH ALGEBRAS: \\ ON SOME GELFAND-MAZUR LIKE THEOREMS \\ IN $P$-NORMED ALGEBRAS: \\ CORRIGENDA
}

\author{
V.K. SRINIVASAN
}

REMARK 1. In my paper "On some Gelfand-Mazur like theorems in Banach algebras" [2], I stated as Theorem 3.1 the following result: If $A$ is a complex Banach algebra, which is locally finite and if $A$ is an integral domain then $A$ is isomorphic to $C$. The proof given there is wrong. The mistake occurred when the principal ideal (h) was considered. Certainly (h) is finitely generated as an ideal, but not necessarily as an algebra. However thanks to the following theorem of Heinze [1], the stated Theorem 3.1 of my paper [2] is still correct. Heinze's results states: An associative locally finite algebra which is also an integral domain, over an algebraically closed field is isomorphic to the ground field. The other theorems stated in [2] are correct.

REMARK 2. In joint paper (with Hu Shaing), "On some Gelfand-Mazur like theorems in $p$-normed algebras" [3], we mentioned that the disc algebra $A(D)$ is a Bezout domain. $A(D)$ is only a pre-Bezout domain. The proof of Theorem 3.4 ( $i i)$ is still valid since pre-Bezout domain is adequate to give the result of Theorem 3.4 (ii).

Received 30 January 1981. The author is indebted to Professor Michael von Renteln for pointing out the correction mentioned in the above Remark 2. 


\section{References}

[1] Joachim Heinze, "A remark on a paper of V.K. Srinivasan", Buzl. Austral. Math. Soc. 22 (1980), 153-154.

[2] V.K. Srinivasan, "On some Gelfand-Mazur like theorems in Banach algebras", Bull. Austral. Mati. Soc. 20 (1979), 211-215.

[3] V.K. Srinivasan and Hu Shaing, "On some Gelfand-Mazur like theorems in p-normed algebras", Bull. Austral. Math. Soc. 21 (1980), 211-221.

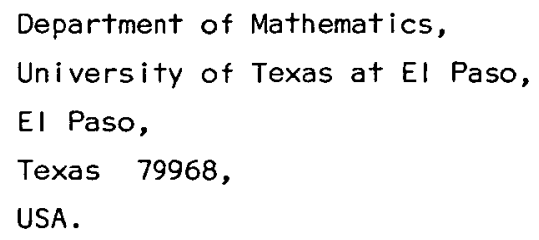

